\title{
Comparison of actuation schemes for wire-driven parallel robots
}

\author{
J-P. Merlet \\ ${ }^{1}$ INRIA Sophia-Antipolis, e-mail: Jean-Pierre.Merlet@inria.fr
}

\begin{abstract}
There are two main systems that can be used to coil and uncoil the wires of a wire-driven parallel robots: a rotary motor that turns a drum on which the wire is coiled or a linear motor with a pulley system. The rotary category may be divided into two sub-categories: the system with a spiral guide for the coiling, allowing only layer for the wire and the system without guide, that allows for several wire layers with the drawback that the amount of coiled wire for one motor turn depends upon the number of layer. All three systems are compared in terms of accuracy and compacity
\end{abstract}

Key words: wire-driven parallel robots, parallel robots, actuation, accuracy

\section{Introduction}

In a wire-driven parallel robot (WDPR) wires are attached at specific anchor points on the robot platform and can be coiled and uncoiled through an actuation system with a fixed output point for the wires. WDPR have been introduced in the 80's [5],[2], as an alternate to parallel robot with rigid links. They share with them the ability to manipulate large load while they allow for larger workspace (as the amount of leg lengths variation is much larger) and present a simpler mechanical design. However their major drawback is that wires can be pulled but not pushed, which increases the complexity of their kinematics as statics has to be taken into account (for example the forward kinematic problem is an open issue [1,8]).

There has been a recent renewal of interest for WDPR in view of new applications: metrology [6], rehabilitation [7, 10], large robot [9], rescue operation $[11,4]$ to name a few.

A very important point for an efficient control of WDPR is a good estimation of the wire length i.e. the length of the wire between the supposed fixed output point of the wire system and its anchor point on the platform. There are two major possible actuation schemes for wire-driven parallel robot:

- the wire is coiled on a drum that is actuated by a rotary motor. The wire length is estimated through measurement of the motor rotation 
- change in the wire length is obtained by attaching the wire to a linear actuator, possibly through a pulleys system in order to amplify the stroke of the actuator(Figure 1). Wire length is obtained through the displacement of the linear actuator. This actuation scheme has been presented for the first time for our MARIONE-REHAB prototype[3]

The drum system is mechanically simple and compact and allows for large change in the wire lengths. We will distinguish two sub-categories for winches with a drum system:

- classical drum: the wire is free to coil on any part of the drum and there may be several layers of wire (Figure 1). This allows for very large changes in the wire lengths. But a major drawback is that the amount of wire length change for a turn of the motor depends on the way the wire is coiled on the drum and on the number of layer. As the coiling process cannot be controlled this may lead to large error in the estimation of the wire length. Furthermore the maximal available torque of the motor leads to a maximal tension in the wire that is dependent upon the radius of the coiled wire i.e. upon the number of layers on the drum

- drum with a spiral guide: here an auxiliary mechanism ensures that the wire is coiled on a spiral guide on the drum and provides a minimal tension in the wire for that purpose (figure 1). Consequently the coiling process is under control, which allows for a good estimation of the wire length. Such drum is mechanically more difficult to design and they have a single wire layer, thereby decreasing the range of possible wire length changes

The purpose of this paper is to compare the advantages and drawbacks of the presented three actuation schemes in terms of accuracy in the estimation of the wire length and compacity.

\section{Actuation schemes analysis}

\subsection{Drum systems}

Let $d$ be the wire diameter, $r$ the radius of the drum, $m$ the number of layer on the drum and assume that the length of the drum is such that $n$ loops can be coiled on the drum. As the wire coils on the drum along a spiral the total length of wire $L$ than can be coiled on the drum is

$$
L=\sum_{j=1}^{j=m} 2 \pi n \sqrt{(r+d / 2+(j-1) d)^{2}+d^{2} /(2 \pi)^{2}}
$$

However the second term in the square root is small compared to the first term and may be neglected. We get 


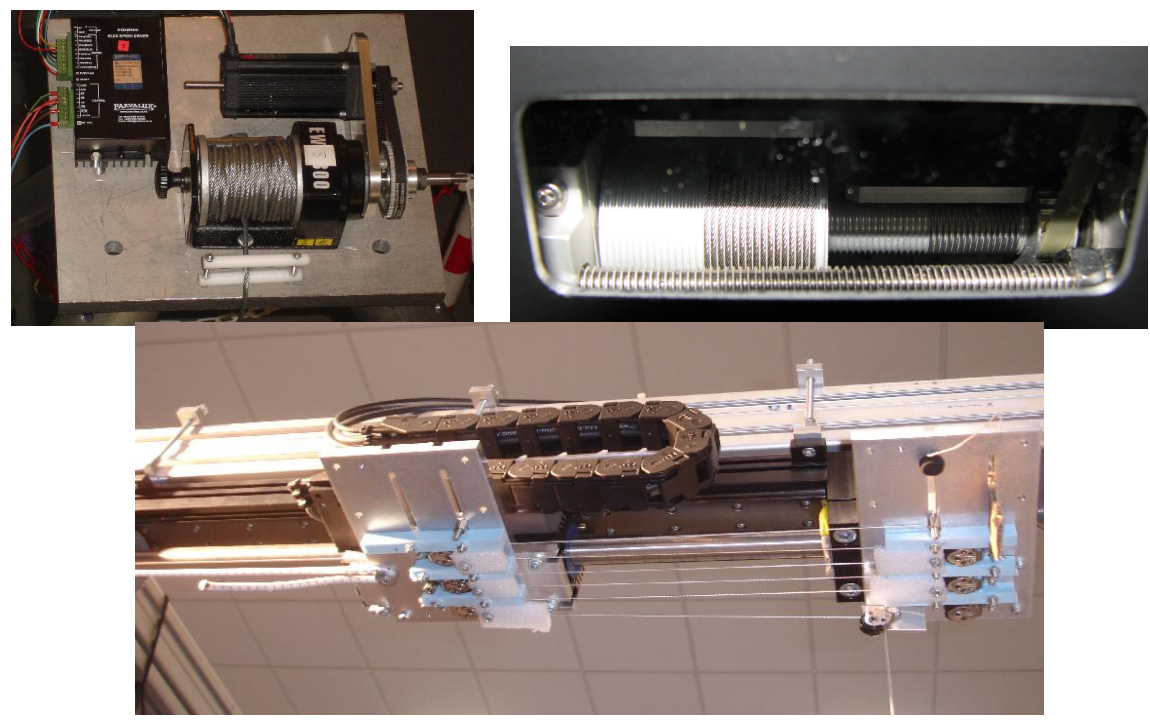

Fig. 1 . On top left the winch of our MARIONET-CRANE robot. On top right the drum of the INCA robot of Haption with a spiral guide on the drum. At the bottom the winch of our MARIONET-REHAB robot which uses a linear actuator and a pulley system.

$$
L=\sum_{j=1}^{j=m} 2 \pi n(r+d / 2+(j-1) d)=2 \pi n\left(m r+d m^{2} / 2\right)
$$

Note that additional effects such as flattening and elongation of the wire are not taken into account because of space restriction. A drawback of the drum system is that the amount of change in the wire length for one motor turn changes according to the number of layer on the drum.

Assuming a perfect coiling, the error $\Delta \rho$ on the wire length estimation for a given error $\Delta \theta$ on the motor rotation angle is:

$$
\Delta \rho=(r+d / 2+(m-1) d) \Delta \theta
$$

where $m$ is the number of layer on the drum. Note also that the maximal available tension $\tau_{\max }$ in the wire for a given maximal torque $\Gamma$ of the motor will change according to the number of layer as:

$$
\tau_{\max }=\frac{\Gamma}{(r+d / 2+(m-1) d)}
$$




\subsection{Linear actuator}

As for the actuation scheme with the linear actuator we have used it for our robot MARIONET-REHAB [3]. If $S$ denotes the stroke of the actuator and $n$ the amplification factor of the pulleys system, then the maximal variation for the wire length is $n S$, the error $\Delta \rho$ on the wire length estimation for a given error $\Delta \theta$ on the actuator pose is $n \Delta \theta$. If $F_{\max }$ is the maximal force of the actuator, then the maximal tension in the wire is $F_{\max } / n$ if we assume no friction in the pulleys. However as such friction exists the amplification factor remains limited. Regarding accuracy a large advantage of this actuation scheme is that accuracy is not affected by the coiling process. Accuracy of linear actuator can be high: as an example we use for MARIONET-REHAB Copley Motion linear actuator with a stroke of $40 \mathrm{~cm}$, an accuracy of $1 \mu \mathrm{m}$ and a pulleys system with $n=10$, leading to an accuracy in the wire length of about $10 \mu \mathrm{m}$, an accuracy that is very difficult to reach with a drum system. Furthermore the amplification system allows to reach very high speed: in MARIONET-REHAB the maximal velocity of the actuator is $10 \mathrm{~m} / \mathrm{s}$ allowing for a wire velocity of $100 \mathrm{~m} / \mathrm{s}$. On the other hand this system offers lower maximal tension in the wire than the drum system and is less compact, while it allows for a higher modularity: a simple change in the number of pulleys allows to modify the workspace, accuracy, velocity and maximal tension in the wire.

\subsection{Comparison between the actuation schemes}

In this section we will compare the actuation scheme for a typical assistance lifting crane. This crane has to be installed in the ceiling of a $4 \times 4$ meter room with a ceiling height of 3 meters. We consider a $4-1$ robot (i.e. a robot having 4 wires, all being attached at the same point $C$ on the platform) with a wire diameter $d$ equal to $0.4 \mathrm{~cm}$, whose actuator are located at the four corners of the room. The fixed output points of the wire system on the ceiling will be denoted $A_{i}$ and we define a reference system whose origin is the anchor point $A_{1}$ with the $z$ axis being the downward vertical. The anchor points of the system are located at

$$
A_{1}(0,0,0), A_{2}(0,400,0), A_{3}(400,400,0), A_{4}(400,0,0)
$$

\subsubsection{Compacity analysis}

It is trivial to show that for reaching any point in the room the maximal wire length $\rho_{\text {max }}$, expressed in $\mathrm{cm}$, should be $\sqrt{400^{2}+400^{2}+300^{2}}=640.31 \mathrm{~cm}$. Using equation (2) it may be shown that for reaching this value the minimal radius of the drum is $\rho_{\max } / 2 m n \pi-d m / 2$. Table 1 presents this radius rounded to centimeter values as a function of the number of loops $(n)$ and number of layers $(m)$. 
For a drum with a spiral guide of pitch $p$ we use equation (1) to determine that the minimal drum radius as a function of the number of loop $n$ is $\sqrt{r o^{2} /(2 n \pi)^{2}-p^{2} /\left(4 \pi^{2}\right)}-$ $d / 2$. Note that the line of $m=1$ of Table 1 is a good approximation of the minimal radius if the pitch is small compared to the drum radius. The drum radius $r$ (in $\mathrm{cm}$ ) is presented for a pitch of $5 \mathrm{~mm}$ as a function of the number of loops $(n)$ in Table 1.

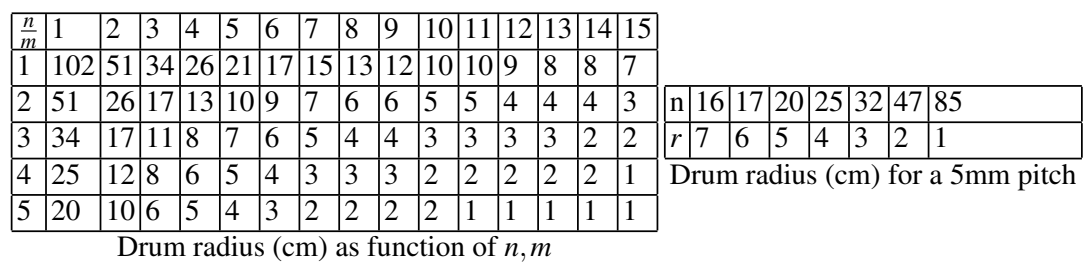

Table 1 Minimal drum radius in $\mathrm{cm}$ to obtain a coiled wire length of at least $640.31 \mathrm{~cm}$ as a function of the number of loops $(n)$ and number of layers $(m)$ for a drum without guide and as function of $n$ for a $5 \mathrm{~mm}$ pitch guided drum

A possible way to compare the two drum actuation schemes is to look at the volume of the drum as it indicates how bulky the system will be. In both case this volume decreases when $m$ (number of layer) and $n$ (number of loop) increase. But the drum with a guide leads in general to a more bulky system: for example such a drum with 47 loops has a volume of $346.6 \mathrm{~cm}^{3}$ while a drum without guide with 3 layers and 10 loops has a volume of $200.75 \mathrm{~cm}^{3}$ while with 4 layers and 9 loops the volume is $149 \mathrm{~cm}^{3}$.

By essence wire system with linear actuator are bulky. They cannot be compared with drum system. Note however that they may be a full part of the frame supporting the robot, thereby being less intrusive.

\subsubsection{Accuracy analysis}

We will reduce our analysis to the workspace reachable by using only actuators $1,2,4$ (because of the symmetry the analysis will be similar for any other actuator choice), which is defined by $y+x-400 \leq 0$. First of all it is necessary to establish the relation between the positioning errors $\Delta \mathbf{X}$ and the errors on the measurement of the wire lengths $\Delta \rho$. The positioning error vector $\Delta \mathbf{X}$ is defined as the vector constituted of the positioning errors $\Delta x, \Delta y, \Delta z$ of the platform along the $\mathrm{x}, \mathrm{y}, \mathrm{z}$ axis. We may assume errors on the wire length measurements that may summed up in the vector $\Delta \rho$. The relation between $\Delta \mathbf{X}$ and $\Delta \rho$ is given by:

$$
\Delta \rho=\mathbf{J}^{-1} \Delta \mathbf{X} \quad \Delta \mathbf{X}=\mathbf{J} \Delta \rho
$$

where $\mathbf{J}^{-1}$ is a notation for the $3 \times 3$ kinematics inverse jacobian matrix. The ith row $J_{i}^{-1}$ of this matrix is defined by $J_{i}^{-1}=\mathbf{A}_{\mathbf{i}} \mathbf{C} / \rho_{i}$ 
If we use a drum with a guide the relation between a change of wire length $\Delta \rho$ and a change in the rotation of the drum $\Delta \theta$ is

$$
\Delta \rho=r \Delta \theta
$$

where $r$ is the drum radius. For a drum without guide this relation is more complex as the drum radius depends upon the current wire length which is itself dependent upon the pose $\mathbf{X}$. We may use equation (2) to determine the possible wire length $\rho_{c}$ that is coiled on the drum for layer $m$ as:

$$
\rho_{c} \in\left[2 \pi n\left((m-1) r+d(m-1)^{2} / 2\right), 2 \pi n\left(m r+d m^{2} / 2\right)\right]
$$

We define the drum function $\mathscr{D}$ which returns the number of layers for a given length $l$ of coiled wire as:

$$
\mathscr{D}: l \rightarrow m / l \in\left[2 \pi n\left((m-1) r+d(m-1)^{2} / 2\right), 2 \pi n\left(m r+d m^{2} / 2\right)\right]
$$

Consequently the drum radius $r_{l}$ for a given length of coiled wire $l$ is

$$
r_{l}=r+d / 2+(\mathscr{D}(l)-1) d
$$

If $\rho_{\text {max }}$ denotes the total wire length, then the drum radius for a given wire length $\rho$ is therefore $r_{\left(\rho_{\max }-\rho\right)}$ and we have $\Delta \rho=r_{\left(\rho_{\max }-\rho\right)} \Delta \theta$ which is therefore a function of $\mathbf{X}$.

In summary to determine the worst case accuracy for a given $\Delta \theta$ it is sufficient to determine the maximum of $\mathbf{J}(\mathbf{X}) \Delta \rho$ for the drum with a guide and of $\mathbf{J}(\mathbf{X}) \Delta \rho(\mathbf{X})$ for the drum without guide, which is therefore a more complex problem.

We will consider now our test example. The $\mathbf{J}^{-\mathbf{1}}$ matrix is $3 \times 3$ and may be symbolically inverted:

$$
\mathbf{J}=\left(\begin{array}{ccc}
\frac{1}{400} \rho_{1} & 0 & -\frac{1}{400} \rho_{4} \\
\frac{1}{400} \rho_{1} & -\frac{1}{400} \rho_{2} & 0 \\
-\frac{1}{400} \frac{(y+x-400) \rho_{1}}{z} & \frac{1}{400} \frac{y \rho_{2}}{z} & \frac{1}{400} \frac{x \rho_{4}}{z}
\end{array}\right)
$$

For a drum with a guide We may assume that the $\Delta \rho_{i}$ lie in the interval [-1,1] and consequently the maximal positive positioning errors $\Delta x_{\max }, \Delta y_{\max }, \Delta z_{\max }$ are given by:

$$
\begin{gathered}
\Delta x_{\text {max }}=\operatorname{Max}\left(\frac{\rho_{1}+\rho_{4}}{400}\right) \quad \Delta y_{\max }=\operatorname{Max}\left(\frac{\rho_{1}+\rho_{2}}{400}\right) \\
\Delta z_{\text {max }}=\operatorname{Max}\left(\frac{-x \rho_{4}-y \rho_{2}+\rho_{1}(y+x-400)}{400 z}\right.
\end{gathered}
$$

The study of the derivatives of $\Delta x_{\max }$ allows one to establish that the largest error will be obtained at $x=0, y=400, z=-300$, with a value of 2.85. A similar maximal value will be obtained for $\Delta y_{\max }$ at $x=400, y=0, z=-300$. The maximal value of $\Delta z_{\text {max }}$ for a given $z$ is obtained at $x=y=200$ with the value $-\sqrt{80000+z^{2}} / z$ 
(which increases for a negative $z$ when $z$ increases). If we limit the value of $z$ to the range [-100,-300], then the maximal value of $\Delta z_{\max }$ is 3 . Note however that this value increases drastically as soon a $z$ cames close to 0 . The above numbers provide directly the error amplification factors for a wire system with linear actuators while for a drum with guide we get the maximal positioning errors as:

$$
\Delta x_{\max }=\Delta y_{\max }=2.85(r+d / 2) \Delta \theta \quad \Delta z_{\max }=3(r+d / 2) \Delta \theta
$$

For the drum without guide the optimization problem is more complex. Assuming a value of 1 for $\Delta \theta$ we get the positioning errors as:

$$
\begin{gathered}
\left.\Delta x=\frac{\rho_{1} r_{\left(\rho_{\max }-\rho_{1}\right)}+\rho_{4} r_{\left(\rho_{\max }-\rho_{4}\right)}}{400} \quad \Delta y=\frac{\rho_{1} r_{\left(\rho_{\max }-\rho_{1}\right)}+\rho_{2} r_{\left(\rho_{\max }-\rho_{2}\right)}}{400}\right) \\
\Delta z=\frac{-x \rho_{4} r_{\left(\rho_{\max }-\rho_{4}\right)}-y \rho_{2} r_{\left(\rho_{\max }-\rho_{2}\right)}+\rho_{1}(y+x-400) r_{\left(\rho_{\max }-\rho_{1}\right)}}{400 z}
\end{gathered}
$$

For finding the maximum of these functions we have to resort to a numerical procedure, based on interval analysis, that is guaranteed to provide the global maximum. The results are provided in Table 2. Such a table is essential for finding the right

\begin{tabular}{|l|l|l|l|l|l|l|l|l|l|l|l|l|l|l|l|}
\hline$\frac{n}{m}$ & 1 & 2 & 3 & 4 & 5 & 6 & 7 & 8 & 9 & 10 & 11 & 12 & 13 & 14 & 15 \\
\hline $1(r)$ & $(102)$ & $51)$ & $(34)$ & $(26)$ & $(21)$ & $(17)$ & $(15)$ & $(13)$ & $(12)$ & $(10)$ & $(10)$ & $(9)$ & $(8)$ & $(8)$ & $(7)$ \\
$\Delta_{x}$ & 291.34 & 145.95 & 97.49 & 74.69 & 60.43 & 49.03 & 43.33 & 37.62 & 34.77 & 29.07 & 29.07 & 26.22 & 23.37 & 23.37 & 20.52 \\
$\Delta y$ & 291.34 & 145.95 & 97.49 & 74.68 & 60.43 & 49.03 & 43.33 & 37.62 & 34.77 & 29.07 & 29.07 & 26.22 & 23.37 & 23.37 & 20.52 \\
$\Delta z$ & 306.60 & 153.60 & 102.60 & 78.60 & 63.6 & 51.6 & 45.6 & 39.6 & 36.6 & 30.6 & 30.6 & 27.6 & 24.6 & 24.6 & 21.6 \\
\hline $2(r)$ & $(51)$ & $(26)$ & $(17)$ & $(13)$ & $(10)$ & $(9)$ & $(7)$ & $(6)$ & $(6)$ & $(5)$ & $(5)$ & $(4)$ & $(4)$ & $(4)$ & $(3)$ \\
$\Delta_{x}$ & 145.95 & 74.69 & 49.03 & 37.62 & 29.07 & 26.22 & 20.52 & 17.67 & 17.67 & 14.82 & 14.82 & 11.97 & 11.97 & 11.97 & 9.12 \\
$\Delta y$ & 145.95 & 74.68 & 49.03 & 37.62 & 29.07 & 26.22 & 20.52 & 17.67 & 17.67 & 14.82 & 14.82 & 11.97 & 11.97 & 11.97 & 9.11 \\
$\Delta z$ & 154.8 & 79.8 & 52.8 & 40.8 & 31.8 & 28.19 & 22.80 & 19.8 & 19.19 & 16.8 & 16.13 & 13.8 & 13.21 & 13.07 & 10.8 \\
\hline $3(r)$ & $34)$ & $(17)$ & $(11)$ & $8)$ & $(7)$ & $(6)$ & $(5)$ & $(4)$ & $(4)$ & $(3)$ & $(3)$ & $(3)$ & $(3)$ & $(2)$ & $(2)$ \\
$\Delta_{x}$ & 97.49 & 49.03 & 31.92 & 23.37 & 20.52 & 17.67 & 14.82 & 11.97 & 11.97 & 9.12 & 9.12 & 9.12 & 9.12 & 6.27 & 6.26 \\
$\Delta y$ & 97.49 & 49.03 & 31.93 & 23.37 & 20.52 & 17.67 & 14.82 & 11.97 & 11.97 & 9.11 & 9.11 & 9.11 & 9.11 & 6.26 & 6.27 \\
$\Delta z$ & 103.8 & 52.8 & 34.8 & 25.8 & 22.8 & 19.8 & 16.8 & 13.8 & 13.8 & 10.8 & 10.8 & 10.8 & 10.8 & 7.80 & 7.8 \\
\hline $4(r)$ & $(25)$ & $(12)$ & $(8)$ & $(6)$ & $(5)$ & $(4)$ & $(3)$ & $(3)$ & $(3)$ & $(2)$ & $(2)$ & 22 & $(2)$ & $(2)$ & $(1)$ \\
$\Delta_{x}$ & 71.83 & 34.77 & 23.37 & 17.73 & 14.82 & 12.12 & 9.61 & 9.31 & 9.12 & 6.76 & 6.64 & 6.50 & 6.35 & 6.27 & 3.99 \\
$\Delta y$ & 71.83 & 34.77 & 23.37 & 17.73 & 14.82 & 12.12 & 9.61 & 9.31 & 9.11 & 6.77 & 6.64 & 6.50 & 6.35 & 6.26 & 3.99 \\
$\Delta z$ & 78 & 39 & 27 & 21 & 18 & 15 & 12 & 11.41 & 11.14 & 9 & 9 & 8.38 & 8.14 & 7.8 & 6 \\
\hline $5(r)$ & $(20)$ & $(10)$ & $(6)$ & $(5)$ & $(4)$ & $(3)$ & $(2)$ & $(2)$ & $(2)$ & $(2)$ & $(1)$ & $(1)$ & $(1)$ & $(1)$ & 1 \\
$\Delta_{x}$ & 58.08 & 29.57 & 18.17 & 15.31 & 12.47 & 9.62 & 6.76 & 6.77 & 6.76 & 6.76 & 4.46 & 4.35 & 4.23 & 4.11 & 3.99 \\
$\Delta y$ & 58.08 & 29.57 & 18.17 & 15.31 & 12.47 & 9.62 & 6.77 & 6.77 & 6.77 & 6.77 & 4.46 & 4.35 & 4.23 & 4.11 & 3.99 \\
$\Delta z$ & 63 & 33 & 21.33 & 18 & 15 & 12 & 9.62 & 9.3 & 9 & 9 & 7.2 & 6.6 & 6.4 & 6.08 & 6 \\
\hline
\end{tabular}

Table 2 Maximal positioning errors of a robot with drums without guide for a rotation error of 1 radian. The errors are presented as a function of the number of loops $(n)$ and number of layers $(m)$. The total wire length is $640.31 \mathrm{~cm}$ and the drum radius is $r$

compromise between bulkiness, accuracy and power of the motors. 


\section{Conclusions}

Winch system is an essential component of a wire-drive parallel robot. Two main winch systems can be considered for wire-driven parallel robots: drums with rotary motor (with or without a spiral track on the drum for guiding the coiling) or linear actuator and pulleys system. The later system leads to the most accurate and fastest winch system but is not appropriate for very large load. Drum with or without guide is the most frequent actuation scheme. We have presented in this paper an in-depth analysis of both systems in term of positioning accuracy.

Acknowledgements The author acknowledges the partial support of the EU through the grant 285404 CableBOT CP-FP

\section{References}

1. Carricato M. and Merlet J-P. Direct geometrico-static problem of under-constrained cabledriven parallel robots with three cables. In IEEE Int. Conf. on Robotics and Automation, pages 3011-3017, Shangai, May, 9-13, 2011.

2. Landsberger S.E. and Sheridan T.B. A new design for parallel link manipulator. In Proc. Systems, Man and Cybernetics Conf., pages 812-814, Tucson, 1985.

3. Merlet J-P. and Daney D. A new design for wire-driven parallel robot. In 2nd Int. Congress, Design and Modelling of mechanical systems, Monastir, March, 19-21, 2007.

4. Merlet J-P. and Daney D. A portable, modular parallel wire crane for rescue operations. In IEEE Int. Conf. on Robotics and Automation, pages 2834-2839, Anchorage, May, 3-8, 2010.

5. Miura K. and Furuya H. Variable geometry truss and its application to deployable truss and space crane arms. In 35th Congress of the Int. Astronautical Federation, pages 1-9, Lausanne, October, 7-13, 1984.

6. Ottaviano E. and Ceccarelli M. Numerical and experimental characterization of singularity of a six-wire parallel architecture. Robotica, 25(3):315-324, May 2007.

7. Perreault S. and Gosselin C.M. Cable-driven parallel mechanisms: application to a locomotion interface. ASME J. of Mechanical Design, 130(10):102301-1/8, October 2008.

8. Pott A. An algorithm for real-time forward kinematics of cable-driven parallel robots. In ARK, pages 529-538, Piran, June 28- July 1, 2010.

9. Riehl N. et al. . On the determination of cable characteristics for large dimension cable-driven parallel mechanisms. In IEEE Int. Conf. on Robotics and Automation, pages 4709-4714, Anchorage, May, 3-8, 2010.

10. Rosati G., Gallina P., and Masiero S. Design, implementation and clinical test of a wire-based robot for neurorehabilitation. IEEE Trans. on Neural Systems and Rehabilitation Engineering, 15(4):560-569, December 2007.

11. Takeda Y. et al. A human body searching strategy using a cable-driven robot with an electromagnetic wave direction finder at major disasters. Advanced Robotics, 19(3):331-347, 2005. 